\title{
Prompt photons at hadron colliders
}

\section{Alessandra Lucà, on behalf of the ATLAS and CDF collaborations *i}

E-mail: aluca@fnal.gov

The production of prompt isolated photons at hadron colliders provides a stringent test of perturbative Quantum Chromodynamics (pQCD), and can be used to probe parton distribution functions (PDFs) and the parton-to-photon fragmentation functions (FFs).

The ATLAS collaboration has performed the measurement of the inclusive production of isolated prompt photons in $20.2 \mathrm{fb}^{-1}$ of data collected at a center-of-mass energy of $8 \mathrm{TeV}$, differential in both pseudorapidity and the photon transverse momentum.

The CDF collaboration has performed the measurement of the cross section for the inclusive production of isolated prompt photons in proton-antiproton collisions at center-of-mass energy $\sqrt{s}=1.96 \mathrm{TeV}$, using the full Run II data sample which corresponds to an integrated luminosity of $9.5 \mathrm{fb}^{-1}$. The cross section is measured as a function of photon transverse energy in the pseudorapidity region $\left|\eta^{\gamma}\right|<1.0$.

The results are compared with predictions from parton-shower Monte Carlo models at leading order in quantum chromodynamics (QCD) and from next-to-leading order perturbative QCD calculations.

38th International Conference on High Energy Physics

3-10 August 2016

Chicago, USA

*Laboratori Nazionali di Frascati, Istituto Nazionale di Fisica Nucleare, I-00044 Frascati, Italy

${ }^{\dagger}$ Speaker. 


\section{Introduction}

The measurement of the inclusive prompt photon $(\gamma)$ production cross section at hadron colliders is an important testing ground for perturbative quantum chromodynamics (pQCD), probing the parton distribution functions (PDFs), and the parton-to-photon fragmentation functions (FFs) $[1,2,3]$.

Inclusive prompt photon production is made up of two contributions: direct and fragmentation photons. An isolation requirement is used to reduce both the poorly understood non-perturbative fragmentation contribution and the contamination from the dominant background of photons originating from hadron decays, mainly light neutral mesons (e.g. $\pi^{0}, \eta$ ).

This proceeding presents recent measurements of prompt-photon production cross section performed by the ATLAS collaboration, using $20.2 \mathrm{fb}^{-1}$ of proton-proton collision data at a centre-ofmass energy of $\sqrt{s}=8 \mathrm{TeV}$, and by the $\mathrm{CDF}$ collaboration, using proton-antiproton collisions at $\sqrt{s}=1.96 \mathrm{TeV}$, based on the full data set and corresponding to an integrated luminosity of $9.5 \mathrm{fb}^{-1}$.

\section{Measurement of the inclusive isolated prompt photon cross section in pp collisions at $\sqrt{\mathrm{s}}=8 \mathrm{TeV}$ with the ATLAS detector}

The dataset used in this analysis [4] was obtained using proton-proton collisions recorded in 2012 by the ATLAS detector, when the Large Hadron Collider (LHC) operated at a centre-of-mass energy of $\sqrt{s}=8 \mathrm{TeV}$, and correponds to an integrated luminosity of $20.2 \mathrm{fb}^{-1}$. The cross section is measured as a function of photon transverse energy, $E_{T}^{\gamma}$, in the range $25<E_{T}^{\gamma}<1500 \mathrm{GeV}$, and in the pseudorapidity regions $\left|\eta^{\gamma}\right|<1.37$ and $1.56 \leq\left|\eta^{\gamma}\right|<2.37$.

\subsection{Photon identification in the ATLAS detector}

The photon candidate in the ATLAS detector [5] is mainly identified by combining the information from the calorimeters and the inner tracker. The events used in the analysis were recorded by the trigger system using single-photon triggers.

The photon selection, in both data and MC simulation, is based on the reconstruction [4] of an electromagnetic (EM) cluster in the calorimeter as a photon candidate. Photons are reconstructed as unconverted (no track in the tracking system) or converted (one or two tracks) photon candidates. In order to suppress hadronic background, photon candidates are then used if they satisfy selection and quality criteria based on their calorimeter shower shapes and isolation energy. Identification criteria have been independently tuned for standard and converted photons.

\subsection{Theoretical predictions}

The theoretical calculations used in the analysis consist of LO Monte Carlo (MC) event generators and calculations at next-to-leading-order (NLO) or higher. Two event generators are used at LO: Pythia 8.165 and SHERPA 1.4.0. These event generators are interfaced with a detailed detector simulation (based on GEANT4), the output of which is reconstructed in the same way as the data. The LO predictions are used to study many aspects of the analysis and are also compared to the final cross section. The final cross sections are also compared to JetPhox, PeTeR, and MCFM calculations. JETPHOX is used as the baseline to compare the results as it is capable of 
calculating the double-differential inclusive prompt photon cross section at parton level to NLO accuracy for both the direct and fragmentation photon processes.

\subsection{Background subtraction}

After the event selection, the hadronic background (from meson decays and jets) is removed by a data-driven technique, which uses a two-dimensional sidebands method based on the isolation and identification criteria. For the identification, photons either satisfy the full criteria of all the showershape variables or an orthogonal selection which aims to maximise the hadronic background. This orthogonal selection is achieved by inverting four variables related to the first layer of the EM calorimeter, which has cells with a very small width in $\eta$. Finally, after the above subtraction, the remaining background of fake photons from electrons is estimated using MC simulations, scaled to the measured integrated luminosity in data, of $Z$ - and $W$-boson decays to electrons.

\subsection{Cross section measurement and uncertainties}

The differential isolated prompt photon cross section, as a function of $E_{T}^{\gamma}$, is calculated in four $\left|\eta^{\gamma}\right|$ regions: $\left|\eta^{\gamma}\right|<0.6,0.6 \leq\left|\eta^{\gamma}\right|<1.37,1.56 \leq\left|\eta^{\gamma}\right|<1.81$ and $1.81 \leq\left|\eta^{\gamma}\right|<2.37$.

An unfolding correction factor is used to correct the measurement to particle level to allow for direct comparisons to theoretical predictions. The unfolding factors are derived using PYTHIA, with SHERPA used as a cross-check, by using a bin-by-bin unfolding procedure.

To estimate the systematic uncertainties, the cross-section calculation was repeated varying the selection procedure, background subtraction techniques or the unfolding correction factor. The main uncertainties to this measurement are the photon energy scale, the admixture between prompt photons $\left(R_{b k g}\right)$ and those coming from fragmentation, and the correlations between the identification and isolation criteria. The energy scale uncertainty dominates the high- $E_{T}^{\gamma}$ region, especially in the region $1.56<\left|\eta^{\gamma}\right|<1.81$. At low $E_{T}^{\gamma}$ the uncertainties from the $R_{b k g}$ variation and admixture of direct and fragmentation photons are of similar magnitude and dominate the uncertainty. In the $E_{T}^{\gamma}$ range $80-200 \mathrm{GeV}$ the main systematic uncertainties are of similar order and, in all but the region $1.56<\left|\eta^{\gamma}\right|<1.81$, this leads to the luminosity uncertainty being larger than this combination of the other systematic uncertainties.

\subsection{Results}

Figures 1, 2 show a summary of the results, where it can be seen that the measurement is well described overall by JETPHOX over ten orders of magnitude in cross section. The results are also compared to LO parton shower MC calculations which shows different trends, with the largest differences being at low $E_{T}^{\gamma}$ in the region dominated by the fragmentation contribution.

\subsection{Conclusion}

In conclusion, a measurement of the inclusive isolated photon cross section has been presented, using $20.2 \mathrm{fb}^{-1}$ of $\sqrt{s}=8 \mathrm{TeV}$ proton-proton collision data recorded by the ATLAS detector at the LHC, spanning $25<E_{T}^{\gamma}<1500 \mathrm{GeV}$, in one of four $\eta^{\gamma}$ regions. The results are compared to JETPHOX predictions, which, for most of the $E_{T}^{\gamma}$ range, have a similar shape but lie below the data. Comparing the results to LO parton shower MC calculations shows different trends, with the largest differences being at low $E_{T}^{\gamma}$ in the region dominated by the fragmentation contribution. 


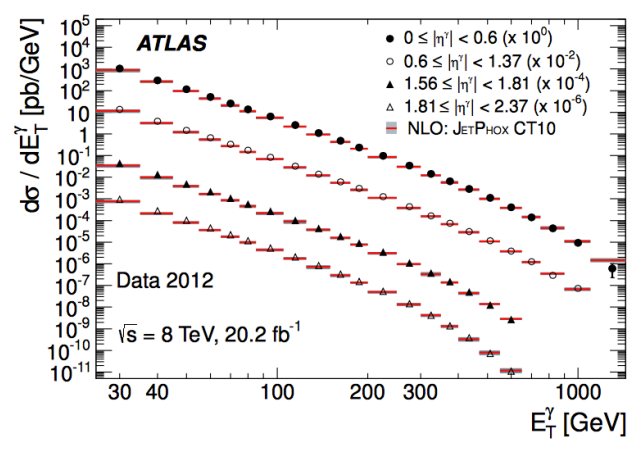

Figure 1: Differential cross sections from data and JетPноX, shown as a function of the transverse energy $E_{T}^{\gamma}$, for the four pseudorapidity $\left|\eta^{\gamma}\right|$ regions. The distributions are scaled, by specified factors.
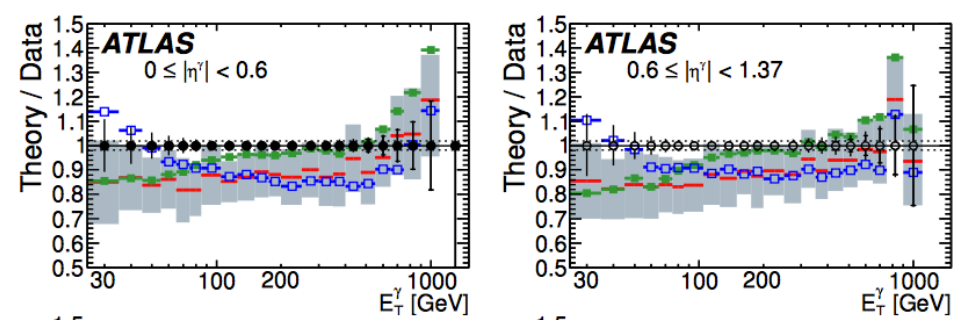

\section{ATLAS}

$\sqrt{\mathrm{s}}=8 \mathrm{TeV}, 20.2 \mathrm{fb}^{-1}$

Data 2012

$0 \leq\left|\eta^{7}\right|<0.6$

$0.6 \leq\left|\eta^{\gamma}\right|<1.37$

$\Delta 1.56 \leq\left|\eta^{\gamma}\right|<1.81$

$\Delta 1.81 \leq\left|\eta^{\Upsilon}\right|<2.37$
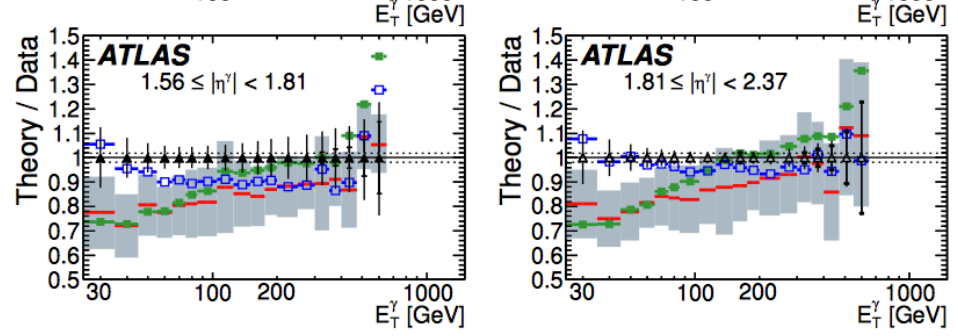

NLO:

$=$ JETPHOX CT10

LO:

口. PYTHIA

$=$ SHERPA

Figure 2: Ratio of theory (PythiA, SherPA and JetPhox) to data for the differential cross sections as a function of the transverse energy $E_{T}^{\gamma}$, for the four pseudorapidity $\left|\eta^{\gamma}\right|$ regions.

\section{Measurement of the inclusive isolated prompt photon cross section in $\mathbf{p} \overline{\mathbf{p}}$ collisions using the full CDF data set}

The data set used in this analysis was obtained using the full Run II data sample collected with the Collider Detector at the Fermilab Tevatron, which corresponds to an integrated luminosity of $9.5 \mathrm{fb}^{-1}$. The cross section is measured as a function of photon transverse energy, $E_{T}^{\gamma}$, in the range $30<E_{T}^{\gamma}<500 \mathrm{GeV}$ and in the pseudorapidity region $\left|\eta^{\gamma}\right|<1.0[6]$.

\subsection{Photon identification in the CDF detector}

The photon candidate in the CDF detector [7] is reconstructed using clusters of (up to three) adjacent towers above threshold in the central EM calorimeter. The data are collected using a three- 
level online event-filtering system (trigger). The photon transverse energy is corrected to account for nonuniformities in the calorimeter response, and calibrated using electrons from reconstructed $Z$-boson decays. Photon candidates are required to satisfy $E_{T}^{\gamma}>30 \mathrm{GeV}$ and to meet requirements on calorimeter isolation. Events with electrons from $Z$ - and $W$-boson decays, which can be misidentified as photons, are removed from the sample by requiring 0 tracks, or at the most one soft track, pointing to the EM cluster. The residual backgrounds from $Z$ - and $W$-boson decays, cosmic rays and beam halo is further reduced to be less than $1 \%$ of the total, by applying additional quality criteria.

\subsection{Theoretical Predictions}

Simulated events from the PYTHIA 6.216 LO MC generator (using the CTEQ5L PDFs), and from the SHERPA 1.4.1 MC generator (using the CT10 PDFs) are produced to study many aspects of the analysis and are also compared to the final cross section. Monte Carlo events are passed through a GEANT-based simulation of the detector and subjected to the same reconstruction and selection requirements as the data. The predicted prompt-photon production cross section is also calculated using the fixed-order next-to-leading-order (NLO) program MCFM 6.8 including nonperturbative fragmentation at LO. This calculation uses the MRST2008 NLO PDFs and the GdRG LO FFs and is corrected for the nonperturbative effects of parton-to-hadron fragmentation and for underlying event energy.

\subsection{Background subtraction}

After the event selection, the remaining hadronic background is removed with a statistical background-subtraction technique. This technique exploits the outcomes of an Artificial Neural Network (ANN), constructed from the shower-shape, transverse profile, and isolation variables. To estimate the prompt-photon rate $\left(f^{\gamma}\right)$, the ANN output distribution observed in data is fit to a linear combination of signal and background ANN templates, constructed using PYTHIA MC predictions. This fit uses a binned maximum-likelihood method that accounts for uncertainties on both data and templates.

\subsection{Cross section measurement and uncertainties}

The differential cross section for the production of isolated prompt photons is calculated as a function of $E_{T}^{\gamma}$ in the central pseudorapidity region $\eta^{\gamma} \mid<1$.0. A correction factor $A \times \varepsilon$, which combines corrections for acceptance, resolution effects and efficiencies for selecting and reconstructing the photon, is used to infer the results at the particle level. The correction is computed from the bin-by-bin fraction of simulated particle-level signal events, as determined by the PYTHIA MC calculation; SHERPA predictions are also used as a cross-check.

The systematic uncertainties on the differential cross section are determined by propagating the sources of uncertainty considered for $f^{\gamma}$ and $A \times \varepsilon$. At low $E_{T}^{\gamma}$ the total systematic uncertainty is dominated by the uncertainties in the ANN-template modeling, while the dependence of the $A \times \varepsilon$ factors on the event generator gives the dominant contribution $(\approx 10 \%)$ to the uncertainty at intermediate and high $E_{T}^{\gamma}$. Finally, there is an additional $6 \%$ uncertainty on the integrated luminosity. 


\subsection{Results}

Figure 3 shows the results compared with the theoretical predictions. The NLO calculations agree with the data up to the highest $E_{T}^{\gamma}$-values considered. Observed cross sections are moderately larger than the central values for the NLO calculation for low $E_{T}^{\gamma}$, but agree within the theoretical uncertainty of the NLO calculation. The shape of the measured-cross-section distribution is well described by PYTHIA and SHERPA predictions. The PYTHIA prediction underestimates the observed cross section by more than a factor of 1.5 across the whole $E_{T}^{\gamma}$ range.
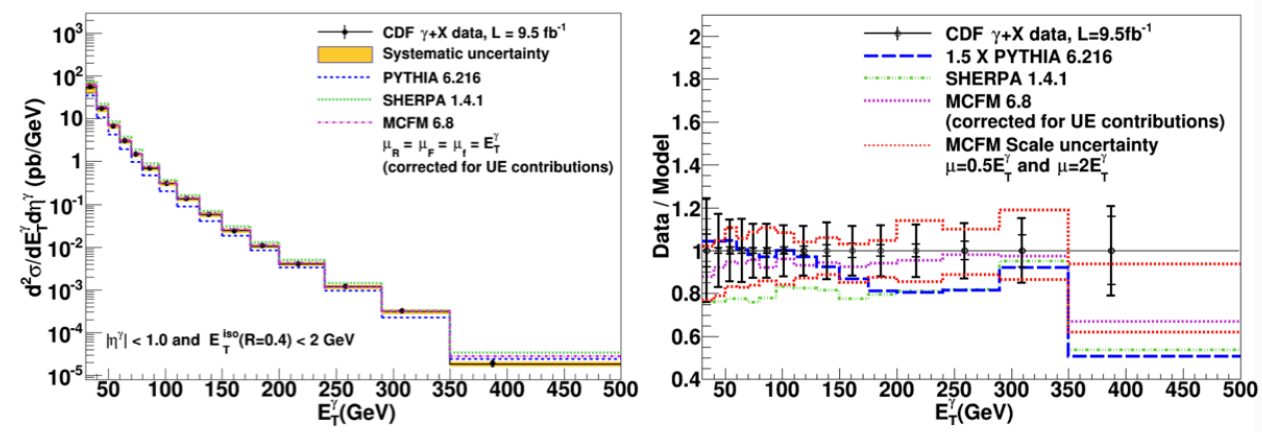

Figure 3: (Left) Differential cross section from data compared with the PYTHIA, SHERPA and MCFM predictions, shown a function of the transverse energy $E_{T}^{\gamma}$, in the pseudorapidity region $\left|\eta^{\gamma}\right|<1.0$. (Right) Data-to-theory ratio (dashed lines); the LO PYTHIA prediction is multiplied by a factor 1.5.

\subsection{Conclusions}

A measurement of the differential cross section for the inclusive production of isolated prompt photons in $p \bar{p}$ collisions at $\sqrt{s}=1.96 \mathrm{TeV}$ is presented using the full data set collected with the CDF II detector at the Tevatron. The cross section is measured as a function of photon transverse energy $E_{T}^{\gamma}$ in the central pseudorapidity region $\left|\eta^{\gamma}\right|<1.0$. The measurement spans the $E_{T}^{\gamma}$ kinematic range from $30 \mathrm{GeV}$ to $500 \mathrm{GeV}$. The data are in good agreement with the fixed-order NLO MCFM calculation. The results are also compared to LO parton shower MC calculations, which have a similar shape but lie below the data.

\section{References}

[1] J. F. Owens, Rev. Mod. Phys. 59, 465 (1987).

[2] W. Vogelsang and A. Vogt, Nucl. Phys. B453, 334 (1995).

[3] L. Bourhis, M. Fontannaz, and J. Guillet, Eur. Phys. J. C 2, 529 (1998).

[4] G. Aad et al. (ATLAS Collaboration), JHEP 08, 005 (2016), 1605.03495.

[5] G. Aad et al. (ATLAS Collaboration), JINST 3 S08003 (2008).

[6] A. Lucà, FERMILAB-THESIS-2016-08

[7] R. Blair et al. (CDF Collaboration), FERMILAB-DESIGN-1996-01, FERMILAB-PUB-96- 390-E (1996). 